Instituto Internacional de Investigación y Desarrollo Tecnológico Educativo INDTEC, C.A. DOI: https://doi.org/10.29394/Scientific.issn.2542-2987.2020.5.16.4.82-98

OAl-PMH: $\underline{\text { http://www.indteca.com/ojs/index.php/Revista Scientific/oai }}$

Artículo Original / Original Article

\title{
Los procedimientos empíricos en el proceso de titulación profesional en la universidad pública
}

Autores: Francisco Manuel Ugarte Palacin Universidad Nacional de Ingeniería, UNI fugarte2006@yahoo.com Lima, Perú https://orcid.org/0000-0002-2129-9342

Ángel Salvatierra Melgar Universidad César Vallejo, UCV ansalme2@gmail.com Lima, Perú https://orcid.org/0000-0003-2817-630X

\section{Resumen}

En el presente estudio se describe la problemática que sucedió en los procesos de titulación por diversas modalidades en la Facultad de Ingeniería Mecánica de la Universidad Nacional de Ingeniería (FIM-UNI) en el periodo 2012-2017, en referencia a la Ley Universitaria 30220. La referida ley pondera las modalidades para obtener el título profesional: primero se debe haber obtenido el grado de Bachiller, así como también haber aprobado una tesis o en todo caso un trabajo de suficiencia profesional, y solamente en aquella universidad en la cual se consiguió el grado de bachiller. Esta investigación ha seguido el método de estudio exploratorio, descriptivo y explicativo; y se aplicó el diseño no experimental transeccional o transversal exploratorio descriptivo. Todas aquellas acciones que contribuyen a la motivación y realización de algún tema de tesis entre la industria y la universidad benefician a los alumnos, lo cual hace que no se sientan ajenos a tal realidad y logren obtener las herramientas adecuadas para experimentar; y de esa manera, evitar ser parte de los empirismos aplicativos.

Palabras clave: tesis; profesional; universidad.

Cómo citar este artículo:

Ugarte, F., \& Salvatierra, Á. (2020). Los procedimientos empíricos en el proceso de titulación profesional en la universidad pública. Revista Scientific, 5(16), 82-98, e-ISSN: 2542-2987. Recuperado de: https://doi.org/10.29394/Scientific.issn.2542-2987.2020.5.16.4.82-98

Fecha de Recepción: 18-11-2019
Fecha de Aceptación: 17-03-2020
Fecha de Publicación: 05-05-2020 
OAI-PMH: http://www.indteca.com/ojs/index.php/Revista_Scientific/oai

Artículo Original / Original Article

\title{
Empirical procedures in the process of professional qualification in the public university
}

\begin{abstract}
This study describes the problem that happened in the titling processes by various modalities in the Faculty of Mechanical Engineering of the National University of Engineering (FIM-UNI) in the period 2012-2017, in reference to University Law 30220. The aforementioned law ponders the modalities to obtain the professional degree: first the Bachelor's degree must have been obtained, as well as having passed a thesis or in any case a work of professional sufficiency, and only in that university in which the degree was obtained Bachelor's degree. This research has followed the method of exploratory, descriptive and explanatory study; and the descriptive nonexperimental transectional or transversal exploratory descriptive design was applied. All those actions that contribute to the motivation and realization of some thesis topic between the industry and the university benefit the students, which means that they do not feel oblivious to this reality and obtain the appropriate tools to experiment; and that way, avoid being part of the application empiricisms.
\end{abstract}

Keywords: theses, professional, university.

How to cite this article:

Ugarte, F., \& Salvatierra, Á. (2020). Empirical procedures in the process of professional qualification in the public university. Revista Scientific, 5(16), 82-98, e-ISSN: 2542-2987. Recovered from: https://doi.org/10.29394/Scientific.issn.2542-2987.2020.5.16.4.82-98

Date Received:

18-11-2019
Date Acceptance:

17-03-2020
Date Publication: 05-05-2020 
Artículo Original / Original Article

\section{Introducción}

Según Caballero (2014): se identifica los empirismos normativos al estar ocurriendo que algún reglamento interno que regula tal realidad, institución o empresa, en su enunciación no incorpora, no está de acuerdo a lo actual o no considera un planteamiento teórico que se relaciona directamente.

De acuerdo con Ibarra (2017a): en nuestros días en el Perú, se mide el ser profesional o no mediante la titulación profesional, aquellos que culminan sus estudios en alguna universidad son considerados profesionales solamente si poseen el título profesional. El problema que existe es que en las universidades públicas cada semestre se incrementa el número de egresados que concluyen satisfactoriamente sus estudios académicos en pregrado sin lograr obtener el título profesional en un tiempo prudencial.

Las promociones salientes de egresados han encontrado diversos obstáculos que generan desánimos y demoras en la obtención de su título profesional. Al motivar a los involucrados se evita tener una idea negativa sobre la misma y se logra ubicar al proceso de investigación por tesis como un aporte que satisface al postulante, sus asesores, institución, comunidad y país. Esta problemática genera que en la actualidad exista un bajo número de titulados mediante tesis de las universidades públicas. La tesis, por ser una de las alternativas con menor preferencia por parte de los alumnos que están por titularse, de forma progresiva se advierte a la tesis como un castigo para aquellos que no cumplen con los requisitos para titularse por otra modalidad.

Señalando a Medina, Watanabe y Angulo (2018): si los postulantes a una universidad pública al elegir la profesión a estudiar tienen varias opciones de elegir, por supuesto que eligen como primera opción la que desearía estudiar, y es así como un buen porcentaje de ingresantes logra un cupo, pero no en la profesión que era su primera opción, lo cual influye en su rendimiento académico durante sus estudios universitarios, ya que produce que se realicen en más de los diez ciclos establecidos, pero mucho más cuando logran la 
condición de egresados, ya que para elegir y plantear su tema de tesis tienen mucha dificultad para realizarlo. Es por lo que los ingresantes y egresados tienen relación directa con la cantidad de alumnos titulado. Los estudiantes al culminar sus estudios escolares deben escoger la profesión a continuar, si esa decisión no es la correcta y no es de acuerdo con su vocación profesional, en los estudios superiores se notan diversos problemas, como por ejemplo un mal rendimiento académico, hasta el abandono de los estudios.

En la Ley Universitaria de la Superintendencia Nacional de Educación Superior Universitaria (SUNEDU, 2014): se expresa que las demoras en la obtención de un tema de tesis, tienen que ver con la ley universitaria actual, pues nos dice que solamente se podrá lograr alcanzar el Título Profesional si es que se tiene el grado de Bachiller y también haber aprobado una tesis o en todo caso un trabajo de suficiencia profesional; además, el grado de Bachiller se logrará obtener si es que se ha aprobado todos los cursos de pregrado correspondientes a la especialidad, la realización de una labor de investigación y también haber estudiado una lengua de otro país, preferentemente inglés o algún idioma nativo. Es decir, para lograr obtener el título profesional se tiene que realizar dos tesis, una para bachiller y otra para la titulación profesional.

El documento que se menciona, tiene vacíos con respecto a las características, formas y obligaciones sobre la titulación, y deja a criterio de cada universidad implantar la concepción y parámetros que mejor le parezca, así también carece de un vocabulario adecuado o definición transparente que estará sirviendo para unir conceptos en todo el país y evitar las interpretaciones diferentes sobre tesis, tesina y proyecto de investigación, entre otros conceptos relacionados a la titulación profesional por tesis u otra modalidad; sin quitar autonomía a cada universidad .

En este sentido, Ibarra (2017b): señala que a todo ello se suma la falta de conocimientos de las normas operacionales, lo que implica que los alumnos desconocen a partir de qué situación o avance curricular ya pueden escribir y 
desarrollar su tema de tesis. Otro factor que influye en las demoras en la obtención de un tema de tesis, es la relación universidad-empresa.

Mencionando a Ramírez y García (2010): el círculo de innovación es la confluencia de seguridad y unión entre la universidad, empresa y gobierno que participan en los cambios de un país con el fin de alcanzar organizaciones e interfaces objetivas para el impulso de proyectos de investigación que ayuden con el adelanto social y económico. Para Medina (2018): se refleja en las carencias de conocimientos avanzados en los cursos de especialidad; así también la falta de recursos para la elaboración de tesis experimentales en el caso de que los laboratorios no se encuentren en condiciones para desarrollar tales investigaciones. La capacidad de sus recursos humanos y de infraestructura las universidades peruanas aún están analizando, pues se debe tener en cuenta que se está innovando los procesos de aseguramiento de la calidad.

En esa misma línea, Rivera, Espinosa y Valdés (2017): complementan que la predisposición de asesores y docentes para apoyar a los tesistas, está influenciada por diferentes razones, como la organización de la oficina de investigación y otros, sobre todo cuando las líneas de investigación no están definidas. Existen diferentes causas por lo que ocurre ello y que afectan las demoras en la obtención del Título, que la presente investigación tratará de identificar. Es importante sensibilizar y producir en los docentes para que noten en la investigación una acción ordinaria que forma parte de su competencia profesional.

Como dice Ibarra (2017c): el flujo de los procesos para titularse es parte de la realidad problemática a considerar en la presente investigación porque tiene que ver con la predisposición de los revisores asignados para ello, la determinación del asesor más adecuado para asesorar un tema de tesis y el cumplimiento de los plazos o tiempos establecidos por el reglamento de parte de los tesistas y revisores. El propósito no es buscar un único formato para la 
presentación de una tesis, tratando de estandarizar ello, pero debe cumplir con requisitos mínimos; y, es importante considerar en las normas operacionales una propuesta sobre la evaluación de lo que sucede durante la preparación de la tesis y los resultados esperados.

En este sentido, Guerrero (2018): afirma que actualmente los procesos secuenciales para obtener la titulación profesional originan que los estudiantes de los últimos ciclos no inicien el desarrollo de su tesis, y los egresados por diferentes razones descuidan su titulación, lo cual originan demoras en la obtención de un tema de tesis de parte de los egresados de pregrado. Por esa razón, la Gestión del Conocimiento (GC) en el interior de las organizaciones universitarias, admite aprovechar enormemente los recursos verdaderamente para que las personas desplieguen su potencial intelectual sobre una materia específica. Los egresados de las universidades públicas tienen una gran desventaja para lograr la titulación profesional si es que en sus mallas curriculares no están incluidos aquellos cursos que aseguren la preparación del plan de tesis y el desarrollo de la tesis, frente a los egresados de otras universidades que si consideran ello y la mayoría al egresar lo hará con el título o el plan de tesis culminado.

El Sistema Nacional de Evaluación, Acreditación y Certificación de la Calidad Educativa (SINEACE, 2016): expone que la titulación profesional, es lo que hace que se reconozca a nombre de la Nación a un profesional, lo cual lo otorga una universidad después de concluir los requerimientos decretados en la Ley Universitaria y luego de terminar el trámite administrativo correspondiente. En el Perú se considera titulado a aquellos bachilleres que hayan aprobado una tesis o un trabajo de suficiencia profesional. Solamente aquellos que hayan logrado la titulación profesional podrán ejercer su profesión.

Acorde con esto, si se considera que la titulación en la universidad es un proceso de formación, implica que se está cumpliendo con la malla 
Artículo Original / Original Article

curricular, la práctica educativa corresponde al proceso de enseñanzaaprendizaje del estudiante durante su permanencia en la universidad, hasta que concluya con su trabajo de titulación, la práctica profesional, es la relación de toda actividad académica y de las actividades profesionales $\mathrm{O}$ especialidades que se estudian en la facultad, el servicio social corresponde al tipo de profesional que la facultad forma para el servicio a la sociedad y la normatividad corresponde al reglamento de titulación de la universidad y la Ley Universitaria.

En este mismo orden de ideas, López, Albíter y Ramírez (2008): señalan que aquellas instituciones en donde se imparte educación superior, la eficiencia terminal se indica con implicancias cuantitativas, así como cualitativas y fórmulas, por una parte, la relación ingreso-egreso-titulación por generaciones, y por otra, es una formulación de la particularidad del proceso mediante la cual se forman los estudiantes. El proceso de formación profesional termina cuando los estudiantes han logrado la titulación profesional. Por lo que la calidad durante el proceso de los estudios de pregrado en la facultad se puede medir relacionando la cantidad de ingresantes, la cantidad de egresados y la cantidad de titulados por ciclo o por año, lo que corresponde a la eficiencia terminal.

La creación científica está compuesta por el conjunto de publicaciones que se han formado mediante los trabajos relacionados a la investigación que lleva a cabo el docente durante la carrera de la docencia universitaria, la cual se considera para un periodo; es considerada habitualmente como una medida del nivel científico alcanzado por un país. Parte de la producción científica son las tesis desarrolladas y sustentadas por los bachilleres, lo cual ha venido disminuyendo debido a que en su mayoría se titulaba por otra modalidad y no por tesis, y en estos tiempos es más crítico porque las modalidades de titulación no son las de antes.

Según Agudelo (2004): la línea de investigación, es un instrumento 
OAl-PMH: http://www.indteca.com/ojs/index.php/Revista_Scientific/oai

Artículo Original / Original Article

fundamental para situar, ordenar, organizar y conducir las políticas de investigación. Si es que no existen líneas de investigación en una facultad de la universidad no se puede centrar un tema de tesis y complica el proceso de titulación. Parafraseando a Bunge (1979): se entiende por Excelencia Académica el resultado de la mejor calidad posible en los procesos de desarrollo académico, es decir, la excelencia es una cualidad que reconoce el esfuerzo que se realiza para alcanzar niveles de calidad superior en la práctica del desempeño en la universidad, sean estás de docencia, investigación, extensión o servicios, lo que involucra tanto a los protagonistas de la actividad académica (profesores, alumnos, investigadores) como también a los elementos necesarios para crear un ámbito adecuado para su desarrollo (espacio, equipamiento, medio ambiente, etc.). La excelencia académica depende de varios factores, como por ejemplo los trabajos de investigación que buscan resolver problemas del país desarrollados por alumnos y profesores, las publicaciones bibliográficas, entre otros.

Para Caballero (2008): el asesor de tesis es un científico de prestigio, con destreza y con una imagen profesional excelente, que tiene los resguardos y los documentos académicos adecuados para ser considerado como uno de los integrantes que componen la ley y la jerarquía en el terreno del saber en el que se encuentra la materia y la metodología que defiende el diseño de tesis que lleva a cabo un estudiante. En la Facultad de Ingeniería Mecánica de la Universidad Nacional de Ingeniería (FIM-UNI), el asesor de tesis es un profesor ordinario o extraordinario o contratado con experiencia profesional o con investigaciones en el tema y que tiene la capacidad de orientar al tesista y supervisar el desarrollo de la realización de la tesis o del informe y así también ser quién de la conformidad para la sustentación, y podrá ser propuesto por el estudiante o egresado o designado por la Comisión de Grados y Títulos. 
Artículo Original / Original Article

\section{Metodología}

El presente estudio se llevó a cabo en la FIM-UNI en el Perú, e involucra a los estudiantes, profesores, egresados y titulados. Bajo el lema de la UNI, la facultad de ingeniería mecánica tiene como misión en sus estudiantes la formación de ingenieros líderes en cada una de las especialidades en que se forman (mecánica, mecatrónica, naval y mecánica eléctrica), así como también darle las competencias necesarias para que realicen investigaciones, innovaciones y realicen gestiones con valores y sentido humanístico, y de esa manera contribuirán con el desarrollo y la protección del medio ambiente, como también la confirmación de nuestra identificación nacional. Ante esto, Aguirre (2002): manifiesta, si es que la universidad considera el sentido humanístico en la formación de sus estudiantes, entonces los debe formar e impulsar al considerar ello en cada una de sus especialidades (facultades); es decir, siempre se debe considerar al ser humano como el valor central.

Mencionando a Hernández, Fernández y Baptista (2010): se consideró como objetivo la descripción de los procesos de titulación por diversas modalidades en la universidad UNI-FIM, por esa razón se consideró a esta investigación como cuantitativa porque se señaló, en medio de ciertas opciones, empleando dimensiones numéricas para estar estipuladas mediante instrumentos del terreno de la estadística; y además, porque esta investigación se inició por la causa y efecto de las cosas.

Esta investigación se realizó siguiendo el método de estudio exploratorio descriptivo y explicativo. Se consideró exploratorio porque se materializo al considerar en el objetivo una materia escasamente investigada y estudiada; descriptivo, ya que registra las características y formas de personas o grupos, sociedades, transformaciones, u otro fenómeno que se sujete a análisis; y explicativo, porque está considerado para responder por los principios de los acontecimientos y fenómenos en las sociedades; y además guía la explicación de lo que acontece en la sociedad. En esta 
investigación, se aplicó el diseño no experimental transeccional o transversal exploratorio descriptivo. Transversal porque los datos se recabaron en un determinado periodo de tiempo, exploratorio ya que el tema de investigación se ha estudiado.

\section{Resultados}

Los egresados de algunas universidades públicas tienen desventaja para lograr la titulación profesional porque en sus mallas curriculares no están incluidos cursos que conllevan al desarrollo de la tesis para la obtención de título profesional.

Al no haber realizado acciones que contribuyeron a la motivación y realización de algún tema de tesis entre la industria y la universidad, los alumnos se convirtieron ajenos a tal realidad y no lograron obtener las herramientas adecuadas para experimentar en ello, lo cual manifestó parte de los empirismos aplicativos. Los alumnos si no percibieron la especialización en algún campo de su carrera profesional durante sus estudios de pregrado, habrán aprendido de todo un poco pero no se especializaron en algo. En un país si sus profesionales no estuvieron vinculados con la industria durante su formación, tales profesionales bastante esfuerzo habrán realizado para contribuir con su desarrollo.

En una facultad de una universidad si las líneas de investigación no se han definido, los alumnos al haber intentado centrar su tema de tesis habrán demorado demasiado si es que consideraron como referencia las líneas de investigación de toda la universidad, porque la universidad debe haber considerado a todas las especialidades en sus líneas de investigación general. Los alumnos al haberse sentido preocupados por culminar sus estudios y trabajar en busca de recursos económicos, ha producido que dejen de lado la parte académica y no han priorizado su tiempo para realizar la tesis.

Los alumnos al no haber tenido información sobre el reglamento de 


\section{Artículo Original / Original Article}

titulación no se preocuparon en iniciar su tema de investigación o demoraron más tiempo en escribir su tema de tesis, lo cual se debió a que desconocían los protocolos de una tesis, lo que es un tema de tesis, las condiciones en la que debe encontrarse un alumno y ya pueda presentar su tema de tesis, entre otros.

Gráfico 1. Porcentaje de titulados con respecto a ingresantes en la Facultad de Ingeniería Mecánica de la UNI, datos obtenidos de la Oficina Central de Recursos Humanos.

EN PORCENTAJE TITULADOS CON RESPECTO A EGRESADOS

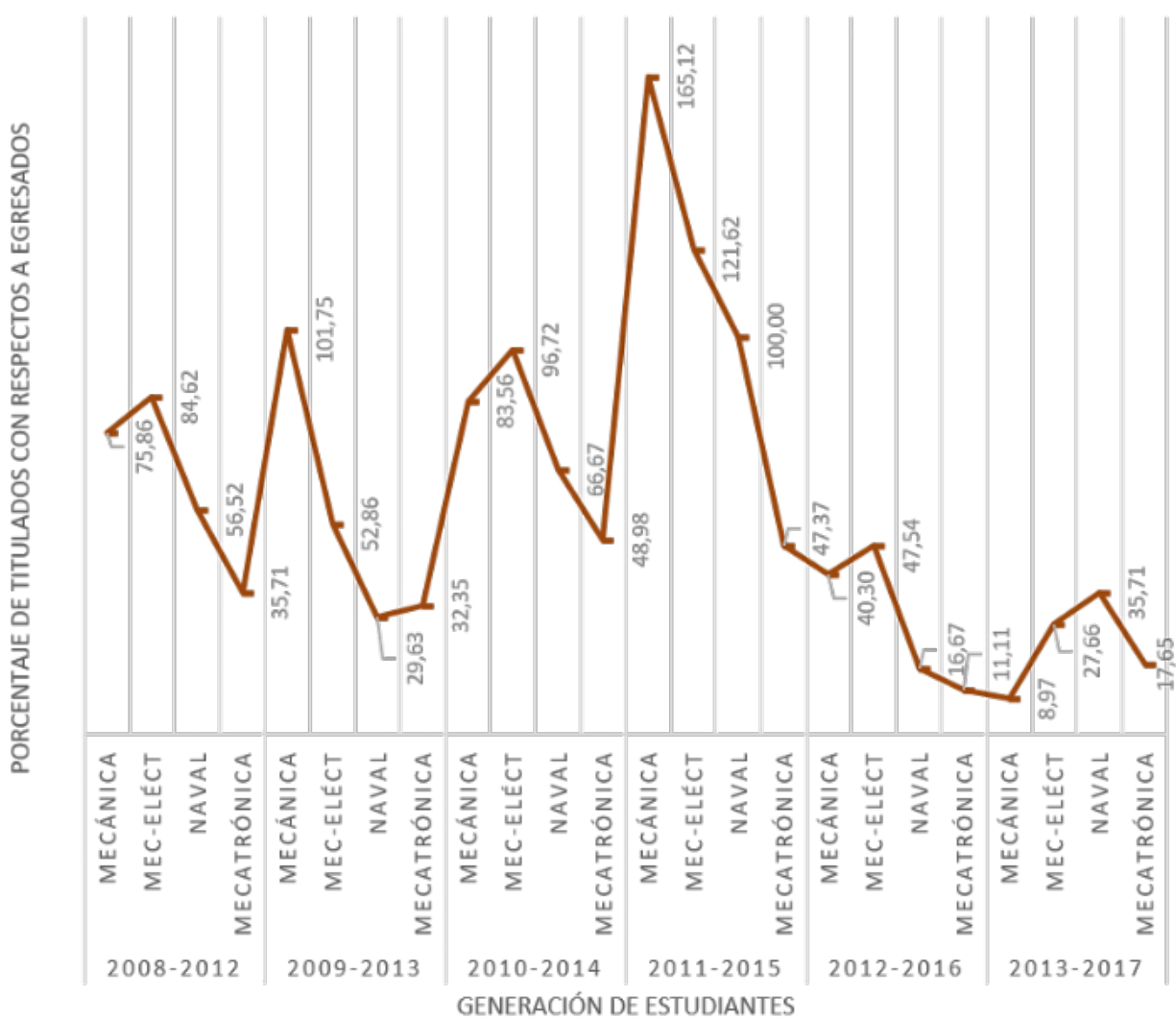

Fuente: Los Autores (2019).

El gráfico 1, fue elaborado en periodos de cinco (5) años, porque toda carrera de la Facultad de Ingeniería Mecánica de la UNI, está programada para 
realizarse en cinco (5) años. Es decir, lo correcto es que los ingresantes a la universidad, deban alcanzar el título profesional después de dicho periodo. Se puede observar, que el porcentaje de titulados ha disminuido después del año 2014, debido a que, desde ese año solamente se puede obtener el título profesional mediante la realización de una tesis o un informe de suficiencia profesional.

Los encargados de la oficina de titulación al no haber utilizado correctamente los conceptos básicos para el proceso de titulación, manifestaron una deficiencia que está relacionada con la falta de la determinación de las líneas de investigación. Una universidad que forma ingenieros, si es que no ha facilitado los laboratorios convenientes a los alumnos, entonces no les permite un desarrollo adecuado, lo cual influye directamente en los procesos de titulación, porque los alumnos encuentran limitaciones en sus investigaciones prácticas durante sus estudios de pregrado, obstaculizando plantear un tema de tesis.

\section{Conclusiones}

Con respecto al objetivo de describir los procesos de titulación, se concluye que está influenciado por el desconocimiento de las normas operacionales por parte de los involucrados. Afirma lbarra (2017d): que al dejar de lado la forma de titulación por tesis se ha recaído en generar conocimiento propio por parte de la universidad, lo que debe de estar comprendido en un espacio-tiempo en la formación de pregrado.

Sobre el flujo de los procesos para titularse los encargados de la oficina de titulación al no emplear bien los conceptos básicos para el proceso de titulación, influye y retarda la inscripción de un tema de titulación. Con relación a eso, Cadena, Escobar y Ortiz (2017), añaden que: Las acciones administrativas se subordinarían a la académica, centraría a la titulación en la exigencia de rigurosidad académica que contempla la formación del 
Artículo Original / Original Article

profesional.

De las demoras en la obtención de un tema de tesis, se identifica que la falta de conocimientos sobre las normas operacionales de parte de los alumnos y bachilleres, los conocimientos avanzados en los cursos de especialidad y las deficiencias de recursos en los laboratorios influye en las demoras de la obtención de un tema de tesis.

Sobre el flujo de los procesos para titularse, se establece que favorece las revisiones de tesis y la facilidad para encontrar un asesor de tesis; en cambio los empirismos aplicativos dificultan tales procesos. Los atrasos en el tiempo para que se culmine una tesis no se deben a los revisores, sino más bien al tiempo que cada tesista le dedica a su investigación. Se recomienda implementar cursos para la realización de la tesis, para bachiller y titulación profesional, e incluyan en su contenido las normas operacionales relacionadas a la titulación.

\section{Referencias}

Agudelo, N. (2004). Las líneas de investigación y la formación de investigadores: una mirada desde la administración y sus procesos formativos. Revista ieRed: Revista electrónica de la Red de Investigación Educativa, 1(1), 1-11, e-ISSN: 1794-8061. Recuperado de: https://dialnet.unirioja.es/servlet/articulo?codigo=2004902

Aguirre, J. (2002). Ciencia, Humanismo, Humanidades y Tecnología. Espéculo: Revista de Estudios Literarios, (19), e-ISSN: 1139-3637. Recuperado de:

https://dialnet.unirioja.es/servlet/articulo?codigo=273569

Bunge, C. (1979). La investigación científica, su estrategia y filosofía. Barcelona, España: Editorial Ariel.

Caballero, A. (2014). Metodología Integral Innovadora para planes y tesis:

La Metodología del cómo Formularios. ISBN: 978-607-519-182-9. 
OAI-PMH: http://www.indteca.com/ojs/index.php/Revista_Scientific/oai

Artículo Original / Original Article

México, D.F.: Cengage Learning Editores S.A. de C.V.

Caballero, A. (2008). Innovaciones en las guías metodológicas para los planes y tesis de maestría y doctorado. ISBN: 978-603-45192-0-6. Lima, Perú: Instituto Metodológico Alen Caro E.I.R.L.

Cadena, S., Escobar, X., \& Ortiz, J. (2017). Unidades de titulación en la universidad central del Ecuador: realidades y retos. Innova Research Journal, 2(8.1), 385-396, e-ISSN: 2477-9024. Recuperado de: https://doi.org/10.33890/innova.v2.n8.1.2017.397

Guerrero, I. (2018). Enfoque Complejo en la Gestión del Conocimiento en Investigación. Revista Scientific, 3(10), 248-266, e-ISSN: 2542-2987. Recuperado de:

https://doi.org/10.29394/Scientific.issn.2542-2987.2018.3.10.13.248$\underline{266}$

Hernández, R., Fernández, C., \& Baptista, P. (2010). Metodología de la investigación. 5ta edición. México: Interamericana Editores, S.A.

Ibarra, P. (2017a,b,c,d). La titulación por tesis en México: el problema de su conceptualización. Dilemas contemporáneos: educación, política y valores, IV(3), 1-22, e-ISSN: 2007-7890. Recuperado de:

https://dilemascontemporaneoseducacionpoliticayvalores.com/ files/20 $\underline{0003500-752 \mathrm{e} 67629 \mathrm{~b} / 17-5-}$

31\%20La\%20titulaci\%C3\%B3n\%20por\%20tesis\%20en\%20M\%C3\%A

9xico.....pdf

López, A., Albíter, Á., \& Ramírez, L. (2008). Eficiencia terminal en la educación superior, la necesidad de un nuevo paradigma. Revista de la educación superior, 37(146), 135-151, e-ISSN: 0185-2760. Recuperado de:

http://www.scielo.org.mx/pdf/resu/v37n146/v37n146a9.pdf

Medina, D. (2018). El rol de las universidades peruanas frente a la investigación y el desarrollo tecnológico. Propósitos y 
OAl-PMH: http://www.indteca.com/ojs/index.php/Revista_Scientific/oai

Artículo Original / Original Article

Representaciones, 6(2), 703-737, ISSN: 2307-7999; e-ISSN 2310-

4635. Recuperado de: http://dx.doi.org/10.20511/pyr2018.v6n2.244

Medina, C., Watanabe, R., \& Angulo, C. (2018). Influencia de la vocación profesional en el rendimiento académico de los estudiantes de medicina veterinaria y zootecnia de una universidad privada de Lima, Perú. RIVEP: Revista de Investigaciones Veterinarias del Perú, 29(4), 1073-1086, ISSN: 1609-9117; e- ISSN: 1682-3419. Recuperado de: https://doi.org/10.15381/rivep.v29i4.15193

Ramírez, M., \& García, M. (2010). La Alianza Universidad-Empresa-Estado: una estrategia para promover innovación. Revista EAN: Escuela de Administración de Negocios, (68), 112-133, ISSN: 0120-8160; e-ISSN: 2590-521X. Recuperado de:

https://doi.org/10.21158/01208160.n68.2010.500

Rivera, C., Espinosa, J., \& Valdés, Y. (2017). La investigación científica en las universidades ecuatorianas. Prioridad del sistema educativo vigente. RECS: Revista Cubana de Educación Superior, 36(2), 113125, e-ISSN: 2518-2730 Recuperado de:

http://www.rces.uh.cu/index.php/RCES/article/view/170

SINEACE (2016). Modelo de Acreditación para Programas de Estudios de Educación Superior Universitaria. Lima, Perú: Sistema Nacional de Evaluación, Acreditación y Certificación de la Calidad Educativa Recuperado de:

https://www.sineace.gob.pe/wp-content/uploads/2014/08/Anexo-1nuevo-modelo-programas-Resolucion-175.pdf

SUNEDU (2014). Ley Universitaria. Ley n 30220. Lima, Perú: Congreso de la República; Superintendencia Nacional de Educación Superior Universitaria. Recuperado de:

https://www.sunedu.gob.pe/wp-content/uploads/2017/04/Leyuniversitaria-30220.pdf 


\section{Artículo Original / Original Article}

\section{Francisco Manuel Ugarte Palacin}

e-mail: fugarte2006@yahoo.com

Nacido en Lima, Perú, el 2 de febrero del año 1962. Maestro

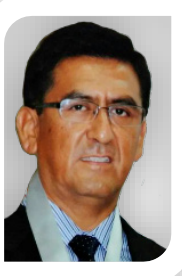
en Ingeniería con mención en Aplicación Energética del Gas Natural, en la Universidad Nacional de Ingeniería (UNI); Ingeniero Mecánico graduado en la Universidad Nacional de Ingeniería (UNI); Actualmente me desempeño como docente universitario en la Facultad de Ingeniería Mecánica de la Universidad Nacional de Ingeniería (UNI). 
OAl-PMH: http://www.indteca.com/ojs/index.php/Revista_Scientific/oai

Artículo Original / Original Article

Natural de Huancayo, Perú, nacido el 20 de mayo del año

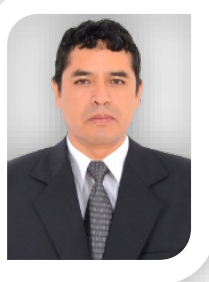
1970. Licenciado en Matemática y Física con grado académico de Doctor en Ciencias de la Educación; Magister en Tecnologías Educativas por la Universidad Nacional del Centro del Perú (UNCP); con diplomatura en Estadística Aplicada por la Pontificia Universidad Católica del Perú (PUCP); docente universitario con 18 años en pregrado en la Universidad Nacional del Centro del Perú (UNCP), Universidad Peruana los Andes (UPLA) y la Universidad Peruana de Ciencias Aplicadas (UPC); Así mismo, docente de postgrado en la Universidad Nacional del Centro del Perú (UNCP) y la Universidad César Vallejo (UCV); Actualmente participo en capacitaciones de Estadística Aplicada para docentes investigadores; Jurado calificador de sustentaciones para la obtención de grado de Magister y Doctor.

El contenido de este manuscrito se difunde bajo una Licencia de Creative Commons ReconocimientoNoComercial-Compartirlgual 4.0 Internacional 